\title{
PULMONARY PSEUDALLESCHERIASIS IN AN IMMUNOCOMPETENT PATIENT - A CASE REPORT
}

Supriya Tankhiwale, Vaishali Rahangdale

1. Associate Professor, Department of Microbiology, Government Medical College,Nagpur.

2. Assistant Professor, Department of Microbiology, Government Medical College,Nagpur..

\section{CORRESPONDING AUTHOR}

Dr. Vaishali Rahangdale, 44, Central Excise Layout, Telecomnagar,Khamla, Nagpur.

E-mail: vaishukolher@gmail.com

Ph: 00918007762999.

ABSTRACT: Psuedallescheria boydii is a common soil fungus that is increasingly reported as a human pathogen. Lung is the most common extra cutaneous site of infections .Invasive pulmonary disease is seen usually in an immunocompromised host.

We are reporting an unusual first case of invasive pulmonary pseudoallescheria sis in an immunocompetent patient from Central India.Microscopy of bronchoalveolar lavage of a treated and cured patient of past pulmonary tuberculosis revealed hyphae (approx 3-5 $\mu$ ) which were septate \& dichotomously branched resembling aspergillus species.The organism was cultured \& identified as P.boydii. . Previously recognized causative agent of disease in an immunocompromised host is now causing disease in immunocompetent host.

KEY WORDS: Pulmonary psuedallescheriasis, Psuedallescheria boydii, cow dung exposure.

MESH TERMS: Pulmonary psuedallescheriasis, Psuedallescheria boydii, cow dung exposure.

INTRODUCTION: Psuedallescheria boydii is a common soil fungus that is increasingly reported as a human pathogen. Lung is the most common extra cutaneous site of infection [1]. Colonization is more common than infection; however an invasive pulmonary disease is seen usually in an immunocompromised host [2].Most of the previously reported cases had prior history of near- drowning and aspiration of polluted water [3]. Here, we are reporting an unusual case of invasive pulmonary pseudallescheriasis in an immunocompetent host in association with a long term history of occupational exposure to cow dung. There are very few reports of such cases from India $\&$ this is the first case of pulmonary psuedallescheriasis from Central India.

CASE HISTORY: A 30 years old female patient from a rural area presented to OPD of respiratory medicine with low grade fever off and on and loss of weight since 9 months, cough with expectoration since 6 months, breathlessness and hemoptysis off and on since 4 months. This patient was having past history of pulmonary tuberculosis treated by short term therapy 3 years back with complete cure. The patient was nondiabetic and nonsmoker. The patient used to do the work of cow dung layering daily in rural households since fifteen years. She did not use any preventive measures during work(e.g. use of masks, covering nose/mouth during work).Patient apparently felt better on nonworking days.

For her present complaints, she received a full course of broad spectrum antibiotic and a four months course of anti-tuberculosis treatment from a private practitioner without any clinico-radiological improvement in the preceding year. The patient remained febrile \& 
symptomatic .So keeping in mind her past history of tuberculosis, she was thoroughly investigated for reactivation of old pulmonary tubercular cavitary lesion.

Her hematological investigations revealed hypochromic macrocytic anaemia with Haemoglobin 9.2 gm\%, TLC - 7,400 / cumm, DLC - PML 63.7 L-23\% M- 14\% E 0.5\% B - 0.5\%, ESR at the end of 1 hour $60 \mathrm{~mm}$. Liver function tests and serum chemistry of the patient was normal. Serological test for HIV was negative. Her CD4 count was within the normal limit. Chest $\mathrm{X}$ - ray (Figure 1) showed nodular shadows predominantly in the right mid zone and lower zone along with homogenous radio opacity in the right lower zone and upper zone suggestive of consolidation. HRCT thorax (Figure 2) was done revealed :1)multiple enhancing parenchymal as well as pleural wall nodular densities with irregular margins and central cavitation involving both lung fields;2)tiny centrilobular nodular opacities involving both lung fields with some of them giving tree in bud appearance ;3)consolidatory changes noted involving apical and posterior segment of right upper lobe, medial segment of right middle lobe with mediastinal lymphadenopathy.Thus the imaging favoured the possibility of an infective etiology. Her sputum sample was sent to microbiology laboratory for aerobic, fungal and mycobacterial examination. Gram stain of sputum showed 1-2 leucocyte / OIF and Gram positive cocci in short and long chains. Ziehl - Neelsen stain of six samples (including two induced samples) of sputum were negative for acid fast bacilli (AFB) in direct smear.

Commensal flora was grown on aerobic culture of sputum. No growth was obtained on mycobacterial and fungal culture of sputum. So bronchoalveolar lavage (BAL) of the patient was sent to Microbiology laboratory for bacterial, mycobacterial and fungal examination. Wet preparation of BAL revealed 2 -3 leucocytes / HPF and fungal hyphae (approx 3-5 $\mu$ in size) which were septate and dichotomously branched resembling aspergillus species. Gram stain of BAL revealed 2 -3 leucocytes/OIF. No organism was seen on Gram stain of BAL. Ziehl - Neelsen l stain of BAL revealed no AFB on direct smear examination. Aerobic, mycobacterial \& fungal culture was done on appropriate media [4].

For fungal culture, specimen was inoculated on Sabouraud's agar and cornmeal agar at 25 degree celsius as well as at 37 degree celsius. After 7 days, characteristic house mouse gray silky colonies with aerial mycelium grew on both these medias. Reverse of the slope was initially white but later become black suggesting a diametaceous fungus (Figure 3).

Lactophenol cotton blue examination of the colony revealed septate hyphae approximately 3 $5 \mu$ in width with long and short conidiophores bearing either single conidia or in small groups. Conidia were oval and typically truncate at their base. Slide culture of this growth was done which again revealed the same morphology as seen on direct microscopy of culture (Figure 4).These tubes were further incubated for 2 to 3 weeks for production of sexual reproductive structures. After 3 weeks Lactophenol cotton blue examination of the colony revealed brown spherical cleistothecia $(100-200 \mu \mathrm{m})$ which was more abundant towards the periphery of culture. These results of morphology on culture, Lactophenol cotton blue examination \& slide culture along with assimilation of urea and glucose was consistent with Psuedallescheria boydii[4]. Sample was again repeated after 8 days and same organism was isolated from the sample.

On clinical, radiological and microbiological grounds it was diagnosed as a case of pulmonary psuedallescheriasis in an immunocompetent patient. Unfortunately we did not tested in- vitro activities of antifungal drugs. Based on medical literature patient was treated with oral itraconazole $200 \mathrm{mg}$ bid for 6 months with close clinico -radiological monitoring. She was also advised of cessation of cow dung exposure and change of work. There was a significant 
clinico-radiological improvement at four months of therapy. She had gained weight and improved appetite with improvement of symptoms along with decrement in radiological shadows(Fig 5).

DISCUSSION: Pulmonary psuedallescheriasis is caused by a ubiquitous fungus Pseudallescheria boydii described as a human pathogen by Saccardo in $1911^{[5]}$. Disseminated disease and pulmonary, sinus, bone, CNS and kidney infections have been described in hosts with a variety of underlying conditions including hematological malignancies, diabetes, immunosuppressive therapy and organ transplant [6].This saprophyte have been isolated from soil, decaying vegetation, poultry and cattle manure, polluted streams and coastal water[7]. Disease due to P. boydii has been infrequently reported and this may reflect problems with its identification. Isolation of P. boydii from sterile sites is diagnostic. Growth of this organism from sputum, BAL, sinus aspirates is convincing of disease when accompanied by hyphae on smears or biopsy [2]. Microbiological diagnosis of P. boydii currently purely depends upon culture \& morphological characterization ${ }^{[8]}$.

Distinguishing P. boydii from Aspergillus is particularly important for adequate treatment as both show similar clinical presentation. P. boydii usually resistant to amphotericin but sensitive to imidazoles \& triazoles in contrast to most of the fungi.

Disseminated psuedallescheriasis in immunosuppressed host has been reported by many authors ${ }^{[9,10]}$. Our case report is unusual as there was no evidence of immunosuppression . The points in favour of diagnosis of fungal lung desease in our patient were 1)history of exposure to cow dung since fifteen years with no preventive measures(so probable route of infection in this case is inhalation);2)radiological picture consistent with an infective etiology;3)Confirmation on fungal culture of bronchoalveolar lavage(twice);4)clinical and radiological improvement shown after antifungal therapy. So as P. boydii infection is clinically similar to other mycosis, culture is required for its diagnosis. And its differentiation from other fungi is particularly important as it is resistant to commonly used antifungal drugs.

To our knowledge this is the first case of pulmonary pseudoallescheriasis to be reported in an immunocompetent host with long term history of occupational exposure to cow dung.As exposure to cow dung is still common in rural areas ,this association could be of significant importance. It should be always kept in mind that previously recognized causative agent of disease in immunocompromised host is now causing disease in immunocompetant host.

\section{REFERENCES:}

1. Rippon J W,Carmichae J W,. Petriellidiosis( allescheriosis):four unusual cases and review of literature, Mycopathologia, 1976; 58: 117 - 124.

2. Nayar R.Fungal infection: Psuedoallescheria boydii. available at http://www.uscap.org/site /iap2006/slides16-5v.htm. Accessed April 28, 2010.

3. Travis LB, Roberts GD, Wilson WR. Clinical significance of Pseudoallescheria boydii: a review of 10 years experience, Mayo Clin Proc, 1985; 60: 531 - 537.

4. Koneman EW, Allen SD, Janda WM, Schreckenberger PC, Winn WC. Color Atlas and Textbook of Diagnostic Microbiology, $5^{\text {th }}$ ed. New York: J B Lippincott Co1997:pp 1006 1015.

5. Grace SM lam,Arthur CWLau,LorettaYC Yam, A women with an unresolving lung infiltrate. Available at http://www.hkresp.com/index .php/clinical-mtg-in-rh/1812004/745. Accessed on september15,2010 
6. Khurshid A, Barnett VT, Sekoson M, Ginzburg AS, Onal E, Disseminated Pseudoallescheria boydii infection in a Nonimmunocompromised host, Chest Journal ,1999; 116(2): 572 - 574.

7. Teng-Yeow Tan, Chia Wei Liou,Ling-Chang Kung, Menengitis caused by Pseudallescheria boydii, Chang Gung Med J, 2004; 27(3): 228-230.

8. Cortez KJ ,Roilides E, Quiroz - Telles F, Meletiadis J, Antachopoulos C ,Knudsen T et al, Infections caused by Scedosporium spp, Clinical Micrbiology Reviews ,2004; 21(1): 157 $-197$.

9. Winston D J, Jordan M C, Rhodes J. Allescheria boydii infection in immunosuppressed host, The Am J of Med, 1997; 63(5): 830-835.

10. Shih L Y, Lee N. Disseminated petriellidiosis (allescheriasis) in a patient with refractory acute lymphoblastic leukaemia, J Clin Pathol,1984; 37: 78 - 82.

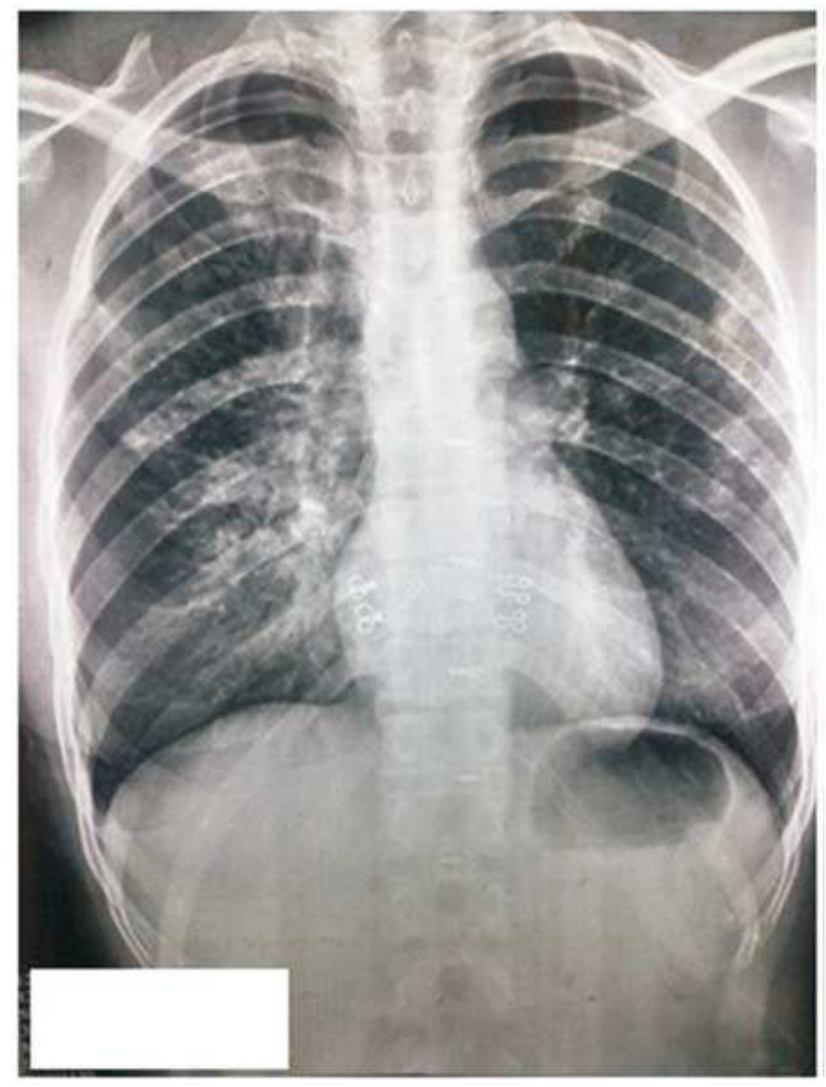

Figure 1.Chest radiograph posterior view at presentation. 


\section{CASE REPORT}

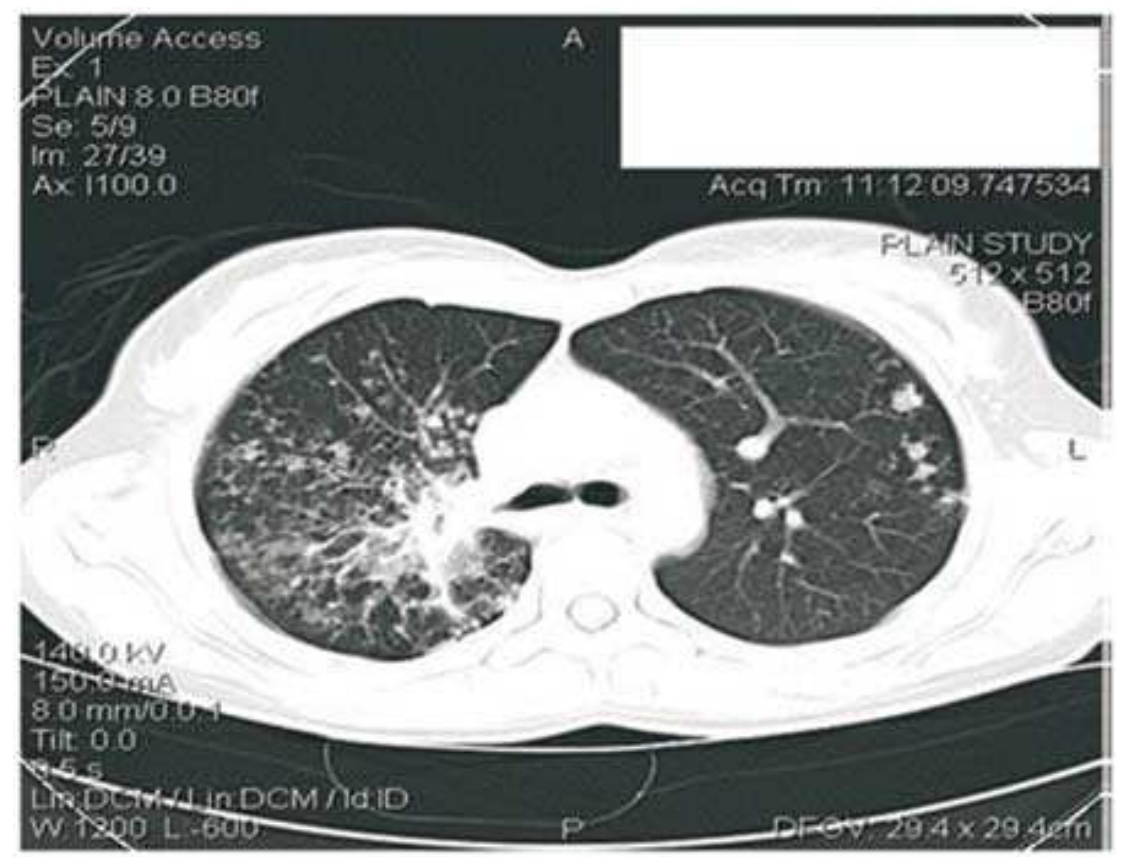

Figure 2.HRCT thorax at presentation

Obverse Side

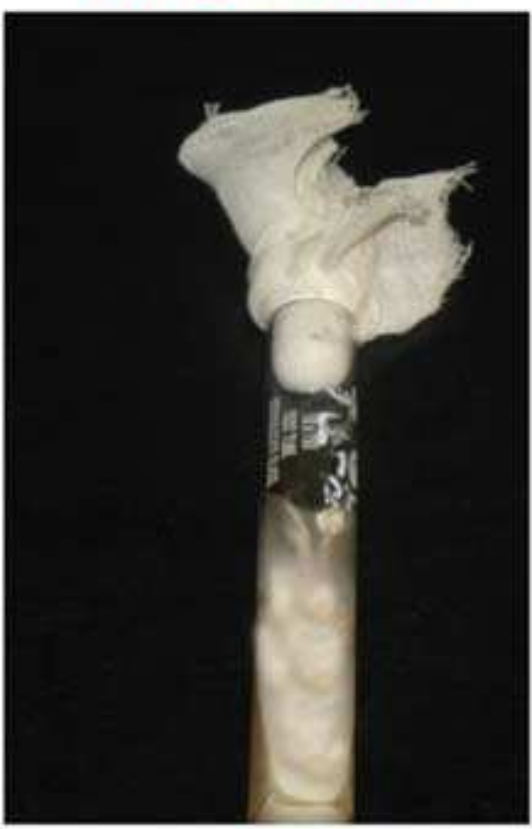

Reverse Side

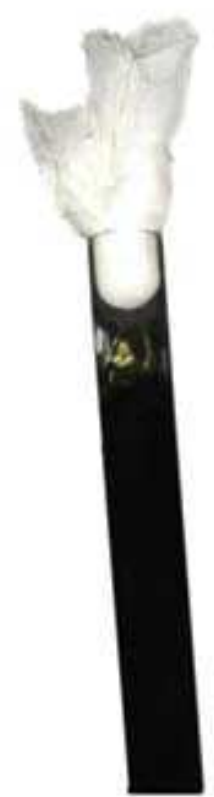

Figure3. Culture of P. boydii on Sabouraud's agar 


\section{CASE REPORT}

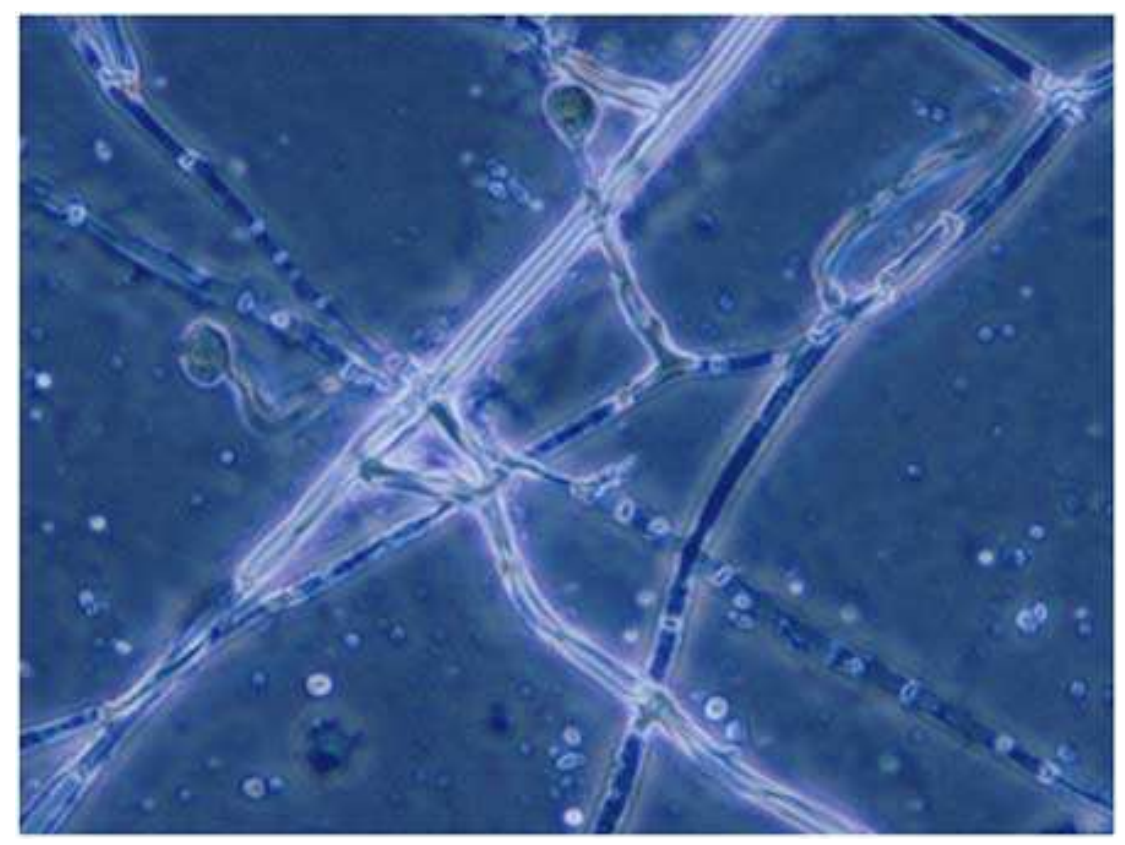

Figure 4. Showing Lactophenol cotton blue preparation of culture

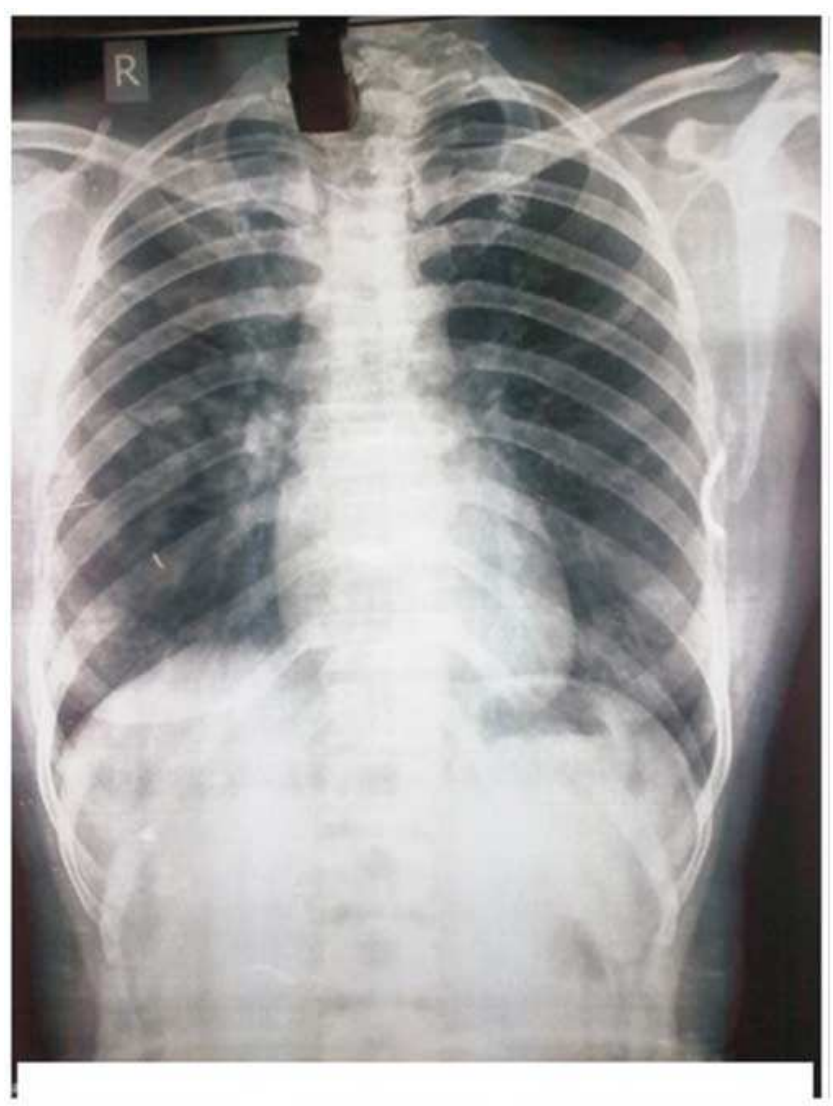

Figure 5.Chest radiograph posterior view after 4 months of itraconazole therapy (significant radiological dissolution). 